\title{
ПОКАЗАТЕЛИ МЕТАБОЛИЗМА ГЛЮКОЗЫ У ЖЕНЩИН С ПРЕДИАБЕТОМ НА ФОНЕ ТЕРАПИИ РАЗЛИЧНЫМИ ДОЗАМИ КОЛЕКАЛЬЦИФЕРОЛА
}

\author{
Андреева' А.Т., ${ }^{2}$ Устюжанина А.О., ${ }^{1,2}$ Каронова Т.Л., 'Гринева Е.Н. \\ 'ФГБУ «Национальный медицинский исследовательский центр им. В.А. Алмазова» МЗ РФ, Санкт-Петербург \\ ${ }^{2}$ ФГБУ «Первый Санкт-Петербургский Государственный Медицинский Университет им. ак. И.П. Павлова» МЗ РФ, \\ Санкт-Петербург
}

ЦЕЛЬ: оценить показатели метаболизма глюкозы на фоне терапии витамином D (колекальциферол) У женщин с предиабетом.

МАТЕРИАЛЫ И МЕТОДЫ: в исследование включены 58 женщин в возрасте 40-55 лет с предиабетом (нарушение толерантности к глюкозе или нарушение гликемии натощак), подписавшие информированное согласие. В исследование не включались женщины, имеющие заболевания, влияющие на метаболизм витамина D, а также получающие регулярную терапию препаратами витамина D. Включенные в исследование женщины рандомизированы на две группы терапии колекальциферолом в течение трех месяцев: Группа I ( $n=30)$ получала терапию в дозе 4000 ME/сут, Группа II $(n=28)-500$ ME/сут. Исходно группы были сопоставимы по возрасту, ИМТ, уровню $\mathrm{HbA}_{1 с}$. При включении в исследование, а также через три месяца терапии колекальциферолом проведена оценка уровня 25(OH)D в сыворотке крови (Abbott Architect $8000, \mathrm{CШA}), \mathrm{HbA}_{1 c^{\prime}}$ концентрация глюкозы плазмы и инсулина в сыворотке крови на фоне проведения ОГТТ $\left(0,60,1200^{\prime}\right)$.

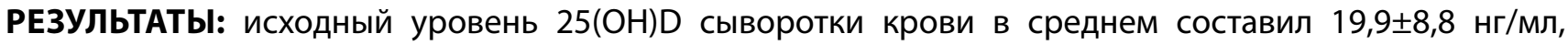
у 30 (51,7\%) женщин выявлен дефицит, у 21 (36,2\%) - недостаток витамина D. Количество лиц с низким уровнем 25(OH)D не отличалось в обеих группах: 28 (93,3\%) и 23 (82,2\%) соответственно. Через 3 месяца терапии колекальциферолом уровень 25(OH)D в сыворотке крови в Группе I составил - 40,4 14,8 нг/мл, в Группе II $-27,8 \pm 10,9$ нг/мл ( $p=0,001)$. Терапия ни в одной из доз не отразилась на показателях ИМТ и ОТ. Анализ параметров метаболизма глюкозы на фоне терапии выявил снижение уровня глюкозы плазмы кро-

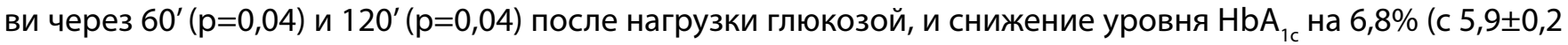
до 5,5 $\pm 0,2 \%)(p=0,001)$ у женщин, получавших колекальциферол в дозе 4000 МЕ/сут. Вместе с тем, терапия не привела к значимому изменению уровня инсулина натощак, но была ассоциирована с повышением

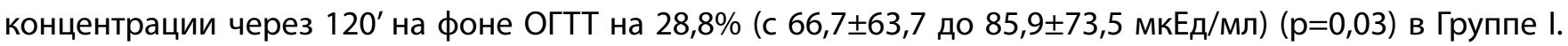
Число женщин, соответствующих критериям предиабета после лечения колекальциферолом в дозе 4000 уменьшилось в два раза. В Группе II (500 ME/сут) не только не было зарегистрировано улучшения показателей гликемии, но и в трех случаях зарегистрированы показатели гликемии, соответствовавшие СД 2 типа.

ВЫВОды: у женщин с предиабетом терапия колекальциферолом в дозе 4000 ME/сут в течение трех месяцев ассоциирована с нормализацией уровня 25(OH)D в сыворотки крови и показателей метаболизма глюкозы.

КЛЮЧЕВЫЕ СЛОВА: витамин D; 25(ОН)D; глюкоза; инсулин; НbА 